\title{
Continuous manufacturing of carboxyamidotriazole- encapsulated nanoemulsions using adaptive focused acoustics: Potential green technology for the pharmaceutical industry
}

\author{
GiHun Choi ${ }^{1}$, Srikanth Kakumanu ${ }^{2}$, Leah Schmitz ${ }^{1}$, Gary LWG Robinson ${ }^{3}$, Carl D. Beckett ${ }^{2}$, James \\ A. Laugharn Jr. ${ }^{2}$ \\ 1. Massachusetts Institute of Technology, Cambridge, USA. 2. Covaris Process Technologies, Covaris Inc., Woburn, USA. \\ 3. RFE Pharma, AL, USA.
}

Correspondence: Srikanth Kakumanu. Address: Covaris Process Technologies, Covaris Inc., 14 Gill St. Unit H, Woburn, MA 01801, USA. Email: skakumanu@covarisinc.com

Received: August 11, $2015 \quad$ Accepted: January 26, $2016 \quad$ Online Published: February 1, 2016

DOI : 10.5430/jbei.v2n2p70 URL: http://dx.doi.org/10.5430/jbei.v2n2p70

\section{Abstract}

Nanoemulsions containing hydrophobic drugs have a great potential in the pharmaceutical industries to improve the bioavailability of the drug. However, currently there is no cost-effective way of producing nanoemulsions in large scale. The need of subjecting emulsions to an extreme pressure of $50 \mathrm{MPa}$ demands a large excess of energy for the manufacturing process, while low-energy method requires large amount of solvents. Here, nanoemulsions containing a well-characterized hydrophobic drug, carboxyamidotriazole (CAI), are produced in both batch and continuous modes to demonstrate the scalability of nanoemulsion production using Covaris' Adaptive Focused Acoustics ${ }^{\mathrm{TM}}$ (AFA) technology. To move from batch scale to continuous flow, the acoustic and thermal energy inputs can be manipulated to adjust particle size, while the composition and temperature of starting materials can be altered to achieve complete dissolution of hydrophobic drugs, thus providing 100\% encapsulation efficiency. Furthermore, using two AFA systems in series can drastically enhance the production flow rates, making AFA a competitive means for producing nanoemulsions in the pharmaceutical industry.

\section{Key words}

Continuous manufacturing, Emulsion, Carboxyamidotriazole, Focused ultrasound, Sonication

\section{I ntroduction}

Nanoemulsions are kinetically stable, but thermodynamically unstable, colloidal dispersions consisting of oil, water, and surfactant in either oil-in-water $(\mathrm{O} / \mathrm{W})$ or water-in-oil $(\mathrm{W} / \mathrm{O})$ form ${ }^{[1-3]}$. For the emulsion to be classified as a nanoemulsion, the droplets in the dispersed phase must have diameters in the nanometric scale, most commonly in the 20 -200 nm range ${ }^{[1]}$. Nanoemulsion shows improvement on the emulsion's transport abilities from larger surface area to volume ratio, thereby increasing bioavailability ${ }^{[4]}$, and resistance to creaming and sedimentation, a frequent dilemma with regular emulsions ${ }^{[5]}$. 
At the same time, many of the advantages of typical emulsions are retained. Both nano and conventional emulsions improve drug solubility, can be manufactured in a fairly large scale, and protect encapsulated drugs from hydrolysis ${ }^{[6]}$.

Delivering drugs within the nanoemulsion droplets confers different favorable properties to the drug. A lipophilic drug encapsulated in an $\mathrm{O} / \mathrm{W}$ nanoemulsion can stay in solution, as nanoemulsion is resistant from creaming ${ }^{[7,8]}$. It will also be easy to produce at a variety of scales, as an abundance of technology exists to produce emulsions in a scalable manner ${ }^{[9,10]}$. As greater than $40 \%$ of new chemical entities developed in the pharmaceutical industry are virtually insoluble in water, while solubility is key for bioavailability, $\mathrm{O} / \mathrm{W}$ nanoemulsions are of particular interest to the pharmaceutical industry ${ }^{[11]}$. Today, O/W nanoemulsions containing hydrophobic drugs are used as anesthetics, NSAID pain relievers, HIV treatments, nutritional supplements, and eye drops, with patents for several more applications filed ${ }^{[7,12-15]}$. There is great potential for an even greater variety of pharmaceuticals as $\mathrm{O} / \mathrm{W}$ nanoemulsions can be delivered via topical, enteral, and parenteral routes ${ }^{[6]}$. However, the drawbacks from nanoemulsion production methods limit which drugs can be encapsulated and commercialized to treat medical conditions ${ }^{[16]}$.

As nanoemulsion is thermodynamically unstable system, it cannot be formed spontaneously without any energy input ${ }^{[1,17]}$. There are two general emulsification methods to deliver the required energy for nanoemulsion production: low-energy and high-energy. The low-energy method explores and utilizes the thermodynamic properties of the materials for emulsification. On the other hand, the high-energy methods use instruments to generate a large amount of energy, whether by high pressure or cavitation, and deliver a small portion of that energy to the sample ${ }^{[1,2,7,8]}$.

The main low-energy methods for producing nanoemulsions are phase inversion temperature (PIT), phase inversion composition (PIC), and solvent displacement. Both PIT and PIC alter the hydrophilic-lipophilic balance (HLB) of a nonionic surfactant, which in turn affects the interfacial properties, by varying easily manipulated system conditions ${ }^{[7,18]}$. This is achieved by changing the temperature in PIT and mixture composition in PIC. Nanoemulsion droplets $\sim 50 \mathrm{~nm}$ in diameter can be produced using these methods ${ }^{[2,7]}$. However, thermolabile compounds cannot be incorporated into the emulsions with PIT, and neither PIT nor PIC can produce nanoemulsions in a scalable manner. Solvent displacement requires large quantities of solvent to dissolve the oily phase in water to spontaneously produce nanoemulsions ${ }^{[7]}$. The amount of solvent dictates particles size, but also creates scale up complications ${ }^{[2,7]}$. It is also important to note that specific combinations of emulsifiers and surfactants are key for successful nanoemulsion using low-energy methods ${ }^{[1,19,20]}$.

Common high-energy modes of nanoemulsion production include high-pressure homogenization, microfluidization, and ultrasonication ${ }^{[1,2]}$. The high-energy methods require less solvent than the low-energy methods, but their excessive energy consumption is a main concern for large-scale production ${ }^{[8]}$. High-pressure homogenizers (HPH) utilize high pressures, typically between 50 and $100 \mathrm{MPa}$, and many forces, such as cavitation, shear, and collision forces. There, microemulsions are accelerated at a high velocity through a tiny opening in the device several times, in order to produce particles as small as $1 \mathrm{~nm}^{[1,2,7]}$. HPH is the most widely used nanoemulsion production instrument, as it offers easy scale up and a fast process time ${ }^{[1,21]}$. At the same time, adding particular type of alcohol to the emulsion mix can enhance the activity of emulsifiers and produce even smaller nanoemulsions via $\mathrm{HPH}^{[22]}$. Nevertheless, HPH's setting and optimized stabilizer conditions are key to reducing drying frequencies for increasing the stability of the nanoemulsions produced ${ }^{[23]}$.

Microfluidization makes use of the high pressure within a microfluidizer. The emulsion mixture is forced through the microfluidizer's microchannels at high pressures, up to $140 \mathrm{MPa}{ }^{[5,7]}$. Exposure to the high pressure accelerates the large droplets to high velocity. The droplets then collide within the interaction chamber with a confined size, which results in a reduction of nanoemulsion sizes ${ }^{[24]}$. Smaller nanoemulsions with narrow distribution could be achieved from the multiple passages through the microfluidizers. However, the absolute size of these droplets is dependent upon emulsion composition, process temperature, and process pressure ${ }^{[2,25]}$. Unlike the typical high-pressure homogenization methods, microfluidization does not require a pre-emulsion step, allowing the immiscible solution to be used in the instrument ${ }^{[26]}$, 
which simplifies the workflow. Despite its advantages, materials used for fabricating the microfluidizer need to be carefully examined. Some materials promote polymer aggregation and swelling on the microchannels, when exposed to organic solvents, that can introduce unfavorable characteristics to the nanoemulsions produced ${ }^{[27]}$. Generating the high-pressure within the microchannels also requires a lot of power. Different fabrication and design strategies, such as stainless steel fabrication and dual microchannels, have been explored to address these issues ${ }^{\text {[26-28] }}$.

Ultrasonic emulsification is unique in that it exploits acoustic field generated by ultrasonicator, deviating from the use of high pressure. The ultrasound applied to initial immiscible solution produces cavitations, small bubbles formed in ambient temperature that then collapse with implosion ${ }^{[8,21,29]}$. Cavitations produce "shockwaves" that introduce turbulence and energy required for nanoemulsion ${ }^{[15]}$. Ultrasound is generated by ultrasonicators, which is then delivered through a large bath of water or tip of the instrument. It has been reported that hydrophobic drugs were successfully encapsulated through ultrasonic emulsification [14, 30-32]. Preparation conditions and instrument settings can be manipulated to yield nanoemulsions with different sizes. Ultrasonic emulsification earns its merit as an energy-saving method, compared to other high-energy methods. It requires 18 times less power for drug encapsulation than the microfluidization into the same size nanoemulsions ${ }^{[15,30]}$. However, current instrumentation and methods require improvements. Bath sonicators are inefficient and inconsistent, as acoustic field is applied to a large bath of water, while the probe sonicators can contaminate samples from directly contacting them ${ }^{[33,34]}$. Although fairly consistent, ultrasonic emulsification using $20 \mathrm{KHz}$ ultrasonic waves yields wider distribution of nanoemulsions than microfluidization, which is unfavorable for the biodistribution ${ }^{[7,15,27,35]}$.

Covaris' patented Adaptive Focused Acoustics ${ }^{\mathrm{TM}}$ (AFA) offers a scalable, precise, sterile, solvent-free, energy-efficient nanoemulsion production method. AFA technology uses focused $500 \mathrm{KHz}-1.1 \mathrm{MHz}$ ultrasonic waves to deliver a specified amount of energy to a focal zone within the solvent-free formulation, creating controlled cavitation. As energy is only being delivered to the sample, not its surroundings, and the system does not need to be brought to high pressures, minimal energy is used. Microjets from imploding cavitation bubbles mill down emulsion droplets to nanoscale dimensions ${ }^{[29]}$. These same microjets mix the formulation, creating a homogenous formulation, and destroy bacteria, sterilizing the mix. The vessel containing the formulation is kept at a constant temperature by a constantly cooled water bath and can take the form of a sealed tube or a flow cell for batch and continuous applications respectively.

In this article, we scale up of production of a simple $\mathrm{O} / \mathrm{W}$ nanoemulsion containing a model hydrophobic compound of pharmaceutical interest: carboxyamidotriazole (CAI). CAI has been pursued as a promising cancer drug for more than 15 years due to the small molecule's anti-angiogenic effects, and has failed numerous clinical studies in the past due to lack of bioavailability ${ }^{[36]}$. We encapsulated CAI in nanoemulsions as an effort to increase its bioavailability. In scaling up CAI nanoemulsion production, parameters such as jacket temperature, flow rate, and energy input, in the form of Peak Incident Power (PIP), were varied to determine the sizes of nanoemulsions that can produced, as well as to create particles of a desired size. Similarly, high levels of encapsulation were achieved in the continuous process by modifying the temperature and composition of the starting materials. In this article, we demonstrate the capacity of AFA technology to produce O/W nanoemulsions containing CAI in a continuous process that exhibits the properties of CAI nanoemulsions produced in batch.

\section{Materials and methods}

\subsection{Nanoemulsions production}

In batch mode, $2 \mathrm{ml}$ of $4 \%(\mathrm{w} / \mathrm{v})$ soybean oil (Sigma-Aldrich, St. Louis, MO) or $4 \%(\mathrm{w} / \mathrm{v})$ rice bran oil (Select Origins, Southampton, NY), 1 mg/ml CAI (RFE Pharma, Framingham, MA), and 4\% (w/v) Tween-80 (Sigma-Aldrich, St. Louis, MO) in water was processed in a $2 \mathrm{ml}$ stainless steel (SST) process vessel from Covaris (Woburn, MA) using an S220x focused ultrasonicator (Covaris) emitting $500 \mathrm{KHz}$ sound waves in a water bath connected to a $3^{\circ} \mathrm{C}$ chiller. Batch samples 
received $45 \mathrm{~kJ}$ of energy per $\mathrm{ml}$ at $300 \mathrm{~W}$ PIP, 50\% duty factor (DF) and 1,000 cycles per burst (C/B). Blank nanoemulsions were prepared following the same procedure omitting the CAI.

For continuous processes, two streams, one consisting of $4 \%$ (v/v) Tween-80 in water and the other consisting of $25 \mathrm{mg} / \mathrm{ml}$ CAI in soybean or rice bran oil, were combined in a 1:25 ratio just before entering a $22 \mathrm{ml}$ jacketed flow vessel from Covaris to form a mix of $4 \%$ (v/v) oil, $1 \mathrm{mg} / \mathrm{ml} \mathrm{CAI}$, and $4 \%$ Tween- 80 in water. The vessel jacket was connected to a $50^{\circ} \mathrm{C}$ chiller. Continuous samples received $9 \mathrm{~kJ}$ per ml of AFA energy at $300 \mathrm{~W}$ PIP, $50 \% \mathrm{DF}$, and 1,000 C/B as the flow rate of the mix was $1 \mathrm{ml} / \mathrm{min}$. Blank nanoemulsions were created according to the same procedure omitting the CAI. Batch and continuous flow setups are depicted in Figure 1.

To evaluate the influence of process parameters on the size distribution of continuously produced nanoemulsions, PIP, flow rate, and jacket temperature were individually varied, keeping the remainder of the production method as described. PIP was set to $200 \mathrm{~W}, 300 \mathrm{~W}, 400 \mathrm{~W}$, and $500 \mathrm{~W}$; flow rate was set to $1 \mathrm{ml} / \mathrm{min}, 2 \mathrm{ml} / \mathrm{min}, 5 \mathrm{ml} / \mathrm{min}$, and $10 \mathrm{ml} / \mathrm{min}$; jacket temperature was set to $3^{\circ} \mathrm{C}, 20^{\circ} \mathrm{C}, 40^{\circ} \mathrm{C}, 50^{\circ} \mathrm{C}$, and $70^{\circ} \mathrm{C}$.

In some continuous flow experiments, AFA processed material from the outlet tube was diluted 100-fold and processed in a second S220x focused ultrasonicator connected in series to the first ultrasonicator. The second processing step was performed in a jacketed $22 \mathrm{ml}$ SST flow process vessel, connected to a $3^{\circ} \mathrm{C}$ chiller, at $300 \mathrm{~W}$ PIP, 50\% DF, and 1,000 C/B in a water bath connected to a $3^{\circ} \mathrm{C}$ chiller.

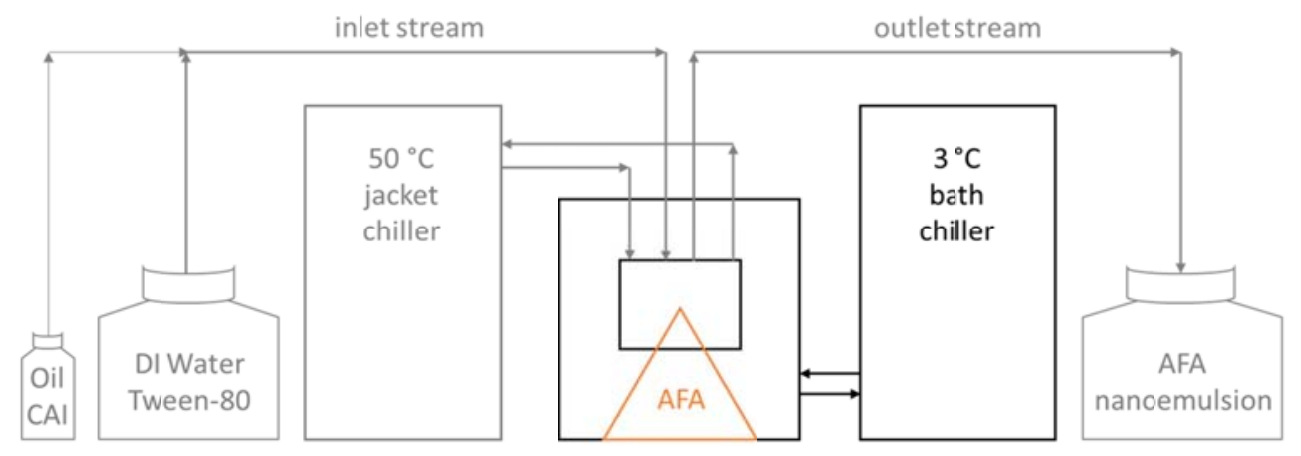

Figure 1. Schematic depicts AFA processing system set up for continuous flow. Oil / CAI stream and water / Tween stream meet before entering flow vessel. Omitting gray portions of diagram reveals batch set up.

\subsection{Particle size distribution determination}

To ascertain the harmonic intensity averaged particle diameter, or z-average size, and polydispersity index, or pdI, of the nanoemulsions, dynamic light scattering (DLS) was used 37 Nanoemulsion samples were diluted appropriately, such that a $1 \mathrm{ml}$ sample in a disposable sizing cuvette from Malvern (Worcestershire, UK) gave an intercept between 0.85 and 0.95 , and analyzed using a Zetasizer Nano ZS90 (Malvern, Worcestershire, UK). Each sample was measured 3 times at $25^{\circ} \mathrm{C}$ using a $90^{\circ}$ scattering angle and the measurements were averaged.

\subsection{CAl concentration verification}

Nanoemulsions were analyzed via a high performance liquid chromatography (HPLC) separations module and $254 \mathrm{~nm}$ absorbance detector (Waters, Milford, MA) to determine CAI concentration in AFA processed samples. Standards were injected onto a Waters (Milford, MA) $4.6 \mathrm{~mm} \times 250 \mathrm{~mm} 5 \mu \mathrm{m}$ xBridge C8 column preceded by a guard column using a 45\% acetonitrile (Thermo Fisher Scientific, Waltham, MA) / 55\% water mobile phase at $1 \mathrm{ml} / \mathrm{min}$. Pressure and temperature were constant at $1,500 \mathrm{psi}$ and $25^{\circ} \mathrm{C}$ respectively. A concentration curve was constructed from DMSO (Sigma-Aldrich, St. Louis, MO) + CAI standards by plotting CAI peak area versus mass of CAI injected and fitting a linear 
model to the data. CAI nanoemulsion samples of $10 \mu \mathrm{l}$ volume were analyzed immediately after the standards under identical conditions. Using the linear model, the concentration of CAI encapsulated in the oil was determined from the sample CAI peak area. Encapsulation efficiency (\%), reported later, is defined as the HPLC CAI concentration divided by the CAI concentration added to the mix times 100.

\subsection{Sterility comparison}

To examine the sterilizing effects of AFA, approximately $1 \mu \mathrm{l}$ of each unprocessed and AFA processed formulation ingredients, including CAI, was plated on both YPD and 7H10 agar plates from Teknova (Hollister, CA) and BD (Franklin Lakes, NJ) respectively. Plates were incubated for 5 days at $37^{\circ} \mathrm{C}$ and then imaged. MALDI Biotyper analysis (Bruker, Billerica, MA) was performed on colonies picked from the plates to determine what kind of contamination, if any, was present in each of the samples. Colonies were picked, resuspended in $50 \mu \mathrm{l}$ water in an AFA microTUBE (Covaris), and centrifuged for 1 minute at 14,000 rpm. The supernatants were discarded and the pellets were resuspended in $33 \mu \mathrm{l}$ water and $97 \mu \mathrm{l}$ ethanol before being centrifuged again at 14,000 rpm for 2 minutes. The supernatants were discarded, the pellets were spun again at the same conditions, and the remainder of the ethanol was removed. $130 \mu \mathrm{l} 70 \%$ formic acid was added before processing the samples in an S220x (Covaris) at $40 \mathrm{~W}$ PIP, 50\% DF, 1,000 C/B for 7 minutes. The samples were transferred to a microfuge tube before $130 \mu \mathrm{l}$ of acetonitrile was added, the samples were vortexed, and the samples were centrifuged for 2 minutes at 14,000 rpm. $1 \mu \mathrm{l}$ of supernatant was spotted onto a 96 target polished steel plate (Bruker) and allowed to dry prior to the addition of $1 \mu \mathrm{l}$ of $10 \mathrm{mg} / \mathrm{ml}$ Bruker HCCA matrix in 2.5\% trifluoroacetic acid, $50 \%$ acetonitrile, and $47.5 \%$ water. Calibration was performed using Bruker E. coli bacterial test standard. Within each sample or standard, 40 locations were analyzed. Acquired spectra were compared to the Bruker BDAL library.

\subsection{Data analysis}

The software Graphpad Prism 6 (GraphPad Software Inc., CA) was used to generate plots for the experimental results. Means and standard deviations were calculated and plotted for the line graphs, whereas the mean values were used to generate the pie charts.

\section{Results}

\subsection{Effect of encapsulating CAI on particle size and distribution}

Nanoemulsions were produced in batch both with and without CAI. HPLC was used to determine CAI concentration in the formulation and $100 \%$ encapsulation $(1 \mathrm{mg} / \mathrm{ml})$ was observed. Particle size distributions were similar for both scenarios (see Figure 2).

Figure 2. Nanoemulsions with CAI (+CAI) produced in batch have a similar z-average and pdI to those without CAI (-CAI) from the same batch method. Each point is the average of 3 replicates.

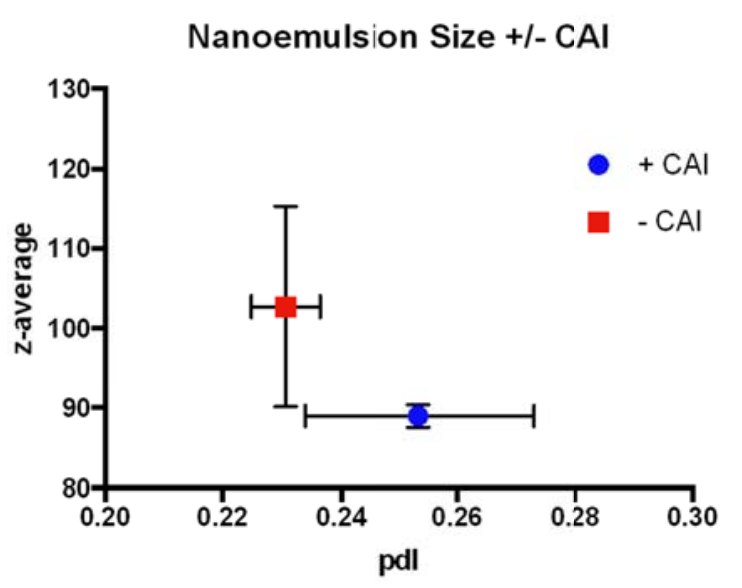




\subsection{Effect of process parameters}

Various process parameters can be examined in this process to determine their effect on the nanoemulsion production. We studied the sample temperature, flow rates and process energy. Decrease in size of nanoemulsions was observed with an increase in PIP values and its effects plateaued with higher power (see Figure 3a). On the other hand, increase in flow rate decreased the residence time of the sample in the process chamber and increased the particle size of the emulsion (see Figure 3b). The nanoemulsions became smaller as we increased the temperature (see Figure 3c).

a)

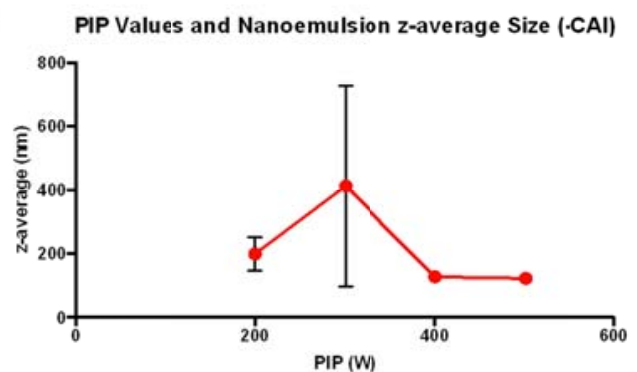

b)

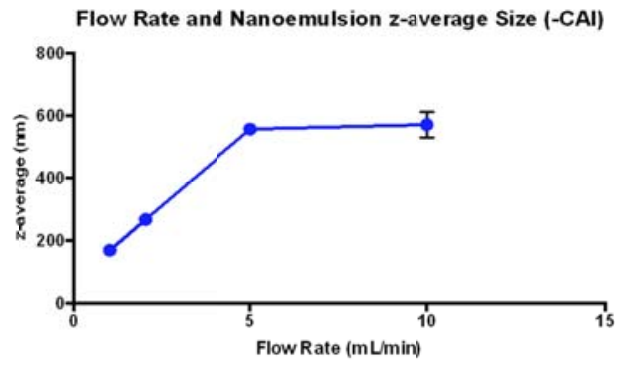

c)

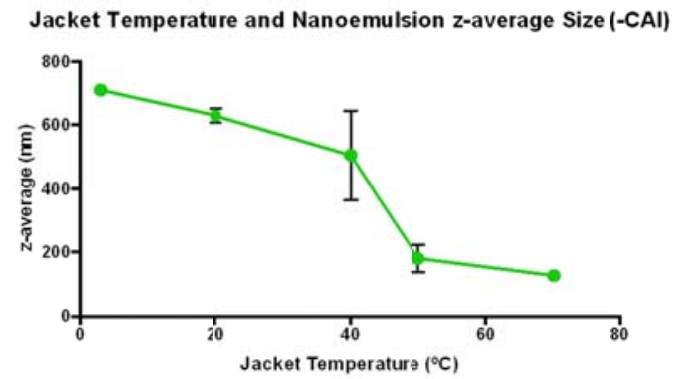

Figure 3. Graphs are scaled separately. All runs lacked CAI. (a) PIP was varied from $200 \mathrm{~W}$ to $500 \mathrm{~W}$. Z-average was measured with DLS after 90 minutes of processing. (b) Flow rate was varied from $1 \mathrm{ml} / \mathrm{min}$ to $10 \mathrm{ml} / \mathrm{min}$. Z-average was measured after more than $90 \mathrm{ml}$ had been processed. (c) Jacket temperature was varied from $3^{\circ} \mathrm{C}$ to $70^{\circ} \mathrm{C}$. Zetasizer readings were taken after 1 hour.

\subsection{Contamination in the samples}

AFA settings with $100 \mathrm{~W}$ PIP or greater are known to lyse bacteria. At $300 \mathrm{~W}$ PIP, formulations are expected to be sterile after AFA processing. To compare the nanoemulsions made in both continuous and batch modes, nanoemulsions produced with both methods, as well as microemulsions (mixture of unprocessed formulation components), were plated on YPD and 7H10 agar plates. Colonies were only observed from the plates streaked with a month old microemulsion (see Figure 4). Performing MALDI-TOF MS on the colonies revealed that the contaminant was a proteobacteria called Cupriavidus sp on both the YPD and 7H10 plates.

\subsection{Batch to continuous production of nano-emulsions}

Nanoemulsions were produced in batch both with and without CAI. HPLC was used to determine CAI concentration in the formulation and $100 \%$ encapsulation was observed. Particle size distributions were similar for both scenarios (see Figure 2). This suggests that the production of nanoemulsions with drugs can be modeled and partially optimized for a target drug using a formulation without the drug.

Individual process parameters were varied in the continuous mode, without CAI, while holding the remaining parameters constant. The parameters varied were PIP, flow rate, and jacket temperature that can all control the amount of energy delivered to the sample: jacket temperature influences the quantity of thermal energy transferred to the formulation, flow rate determines the amount of energy from AFA per ml, and PIP dictates the quantity of AFA energy delivered per burst. 
Zetasizer data revealed a decrease in nanoemulsion size from an increase in both PIP and jacket temperature (see Figure 3). Increasing the temperature decreased the time it took for the process to reach a steady state and produced more consistent particle distributions.

Figure 4. YPD agar plates with $1 \mu \mathrm{l}$ (a) a month-old microemulsion, (b) fresh microemulsion, (c) 2-monthsold batch nanoemulsion and (d) 2-weeks-old continuous emulsion. The plate with a month-old microemulsion has 3 large colonies of Cupriavidus sp (in red oval).
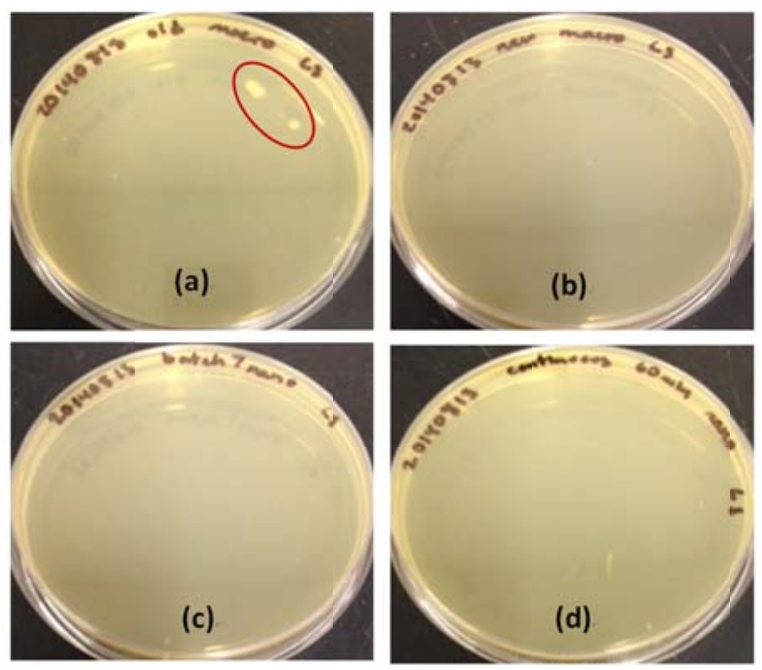

Based on previous results, a combination of parameters was selected to produce nanoemulsions with a z-average below $200 \mathrm{~nm}$. A cutoff of $200 \mathrm{~nm}$ was selected so that the formulation could be easily filtered through a $0.2 \mu \mathrm{m}$ filter to sterilize, which is a common practice in the pharmaceutical industry. At $300 \mathrm{~W}$ PIP, $50 \% \mathrm{DF}, 1,000 \mathrm{C} / \mathrm{B}, 1 \mathrm{ml} / \mathrm{min}, 50^{\circ} \mathrm{C}$ jacket temperature, and $3^{\circ} \mathrm{C}$ bath chiller temperature, nanoemulsions were produced both with and without CAI. Once the processes plateaued, the two nanoemulsions exhibited the similar z-average ( 150 nm) and pdI (see Figure 5). This confirms that optimizing nanoemulsion production before incorporating the drug is a plausible approach. Multiple experiments performed without CAI suggest that it requires 50 minutes to reach steady state level, but nanoemulsions with CAI appeared to do so earlier. Nevertheless, both nanoemulsions had similar size distributions after 50 minutes.

Continuous Processing and Nanoemulsion z-average Size +l- CAI

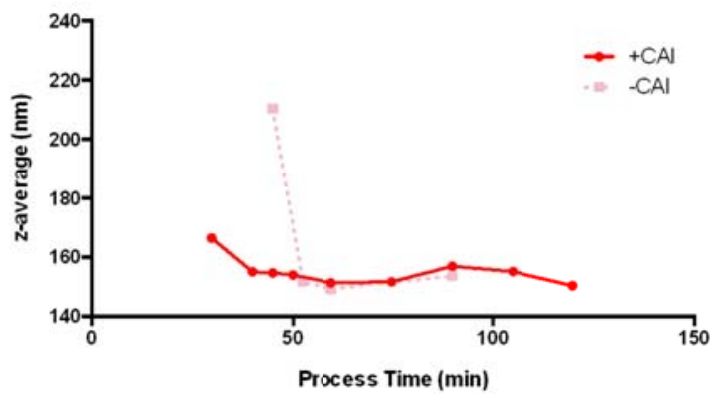

Cotinuous Processing and Nanoemulsion pdl +/-CAl

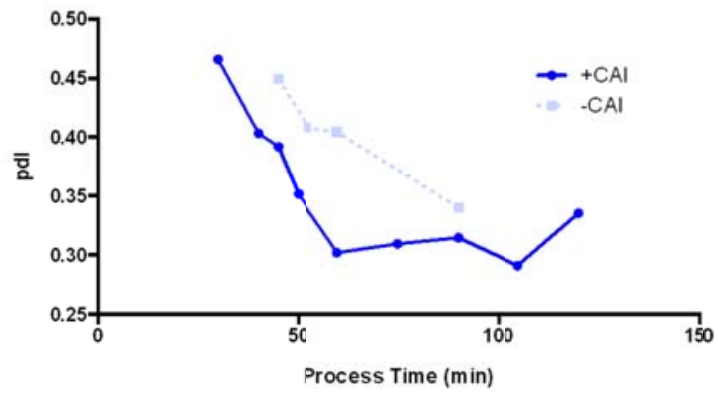

Figure 5. Nanoemulsions were produced with and without CAI under identical conditions in a continuous process mode. Z-average and pdI were measured at time points during the process and are plotted above.

Initially, we aimed to obtain nanoemulsions encapsulating $1 \mathrm{mg} / \mathrm{ml}$ of CAI with a simple oil/CAI mixture in continuous process, in order to examine whether we can achieve the similar encapsulation efficiency as the batch processing. HPLC analysis of CAI concentration in the CAI nanoemulsion revealed only 35\% encapsulation with the continuous process (see Table 1). This was likely due to incomplete dissolution of the drug in the oil, as the oil never clarified after CAI was added and settled when stirring ceased. Having an inhomogeneous suspension resulted in a poor CAI encapsulation. In order to effectively dissolve CAI in oil, we attempted to heat the oil and add other components to the oil/CAI mix. Heating the oil/CAI mix to around $160^{\circ} \mathrm{C}$ still did not dissolve CAI, but did result in $45 \%$ to $76 \%$ encapsulation efficiencies. The 
z-average remained below $200 \mathrm{~nm}$, but did increase by $30 \mathrm{~nm}$. Elevated temperatures changed the color of the oil/CAI mix and melted the tubing.

Table 1. Calculated CAI concentrations from HPLC analysis based on the DMSO standards containing known amounts of CAI

\begin{tabular}{llll}
\hline CAI concentration in continuously processed samples & & \\
\hline Time (min) & Calculated $($ CAI $)(\mathbf{m g} / \mathbf{m l})$ & Experiment & Filtered? \\
\hline 120 & 0.350 & Oil + CAI & No \\
120 & 0.340 & Oil + CAI & Yes \\
45 & 0.466 & Oil + CAI + High Heat & No \\
60 & 0.449 & Oil + CAI + High Heat & No \\
75 & 0.763 & Oil + CAI + High Heat & No \\
75 & $0.765^{*}$ & Oil + CAI + High Heat & Yes \\
90 & Oil + CAI + High Heat & No \\
50 & 0.650 & Oil + CAI + Tween + Heat & No \\
60 & 1.854 & Oil + CAI + Tween + Heat & No \\
60 & Oil + CAI + Tween + Heat & Yes \\
90 & 1.894 & Oil + CAI + Tween + Heat & No \\
120 & 1.179 & Oil + CAI + Tween + Heat & No \\
120 & 1.808 & Oil + CAI + Tween + Heat & Yes \\
\hline
\end{tabular}

Note. Starred $(*)$ value indicates the sample that was ran in a different HPLC run with repeat sample and was scaled with respect to the repeat samples to provide a better comparison within the experiment.

Next, Tween-80 was included in the oil/CAI mix, instead of DI water, to halve the necessary concentration of CAI in the mix and aid in dissolving the drug. CAI effectively dissolved and HPLC analysis of the samples showed $185 \%$ encapsulation from 30 to 120 minutes, suggesting that more CAI was entering the nanoemulsion system than anticipated $(1 \mathrm{mg} / \mathrm{ml})$. The z-average was reduced to $\sim 135 \mathrm{~nm}$, staying below the $200 \mathrm{~nm}$ cutoff. Two distinct phases were apparent in the mix if it was allowed to settle, but stirring maintained a homogenous mix. Both heated oil and Tween-80 experiments had ramp times of approximately 50 minutes, supporting the notion that the short ramp time reported earlier was not a complete representation of continuous CAI nanoemulsion production. The encapsulation efficiency progression suggests that getting the drug into the flow vessel is the limiting factor. Covaris AFA will encapsulate $100 \%$ of hydrophobic drugs in $\mathrm{O} / \mathrm{W}$ nanoemulsions in both batch and continuous modes, up until a certain saturation concentration.

Although AFA processing can bring sterilization effects, sterile filtration was performed on the nanoemulsions. This is the standard procedure in a drug formulation for the pharmaceutical industries that the effect of filtration on the CAI nanoemulsion yields was observed. HPLC was used to analyze the loss of CAI to filtration. In $1 \mathrm{mg} / \mathrm{ml} \mathrm{CAI}$ nanoemulsions produced in batch with an original z-average of $75 \mathrm{~nm}, 98 \%$ of CAI was retained. $62 \%$ of CAI was retained in $1.85 \mathrm{mg} / \mathrm{ml}$ CAI nanoemulsions produced continuously with an original z-average of $135 \mathrm{~nm}$, such that the final concentration was $1.17 \mathrm{mg} / \mathrm{ml}$, or approximately the maximum encapsulation concentration. Particle size distributions of the filtered samples were also determined. The z-averages for filtered batch and continuous emulsions were approximately $37 \mathrm{~nm}$ and $46 \mathrm{~nm}$ respectively (see Figure 6). Volume breakdowns of Zetasizer data revealed a reduction in $5 \mu \mathrm{m}$ droplets in both samples. In the batch processed sample, these particles were eliminated, while over two thirds of the $5 \mu \mathrm{m}$ particles were eliminated for the continuously processed sample. Differences could be due to the discrepancy in initial z-average. However, it is clear that both versions respond well to filtration.

In addition to successful filtration on both formulations, they show stability over time at room temperature (see Table 2). The batch samples were monitored for 2 months. A slight decrease in particle size occurred within the first 24 hours, but the size was remarkably consistent afterwards. Continuous samples were also monitored for 2 months and the original measurement was followed by constant z-average values. 
Table 2. Measured size of nanoemulsions produced by both batch and continuous production methods. In both cases, observed sizes tend to decrease minimally in the first 24 hours, before plateauing and remaining constant

\begin{tabular}{llllll}
\hline \multirow{2}{*}{ Time since production } & Batch & & & Continuous \\
\cline { 2 - 3 } \cline { 5 - 6 } & z-average (nm) & pdl & & z-average (nm) & 0.441 \\
0 Day & 71.62 & 0.324 & 123.70 & 0.431 \\
1 Day & 66.47 & 0.310 & 121.70 & 0.473 \\
1 Day Month & 66.81 & 0.311 & 121.20 & 0.463 \\
2 Month & 66.37 & 0.296 & 122.30 & 0.465 \\
\hline
\end{tabular}

(a) Unfiltered Batch CAI Processing

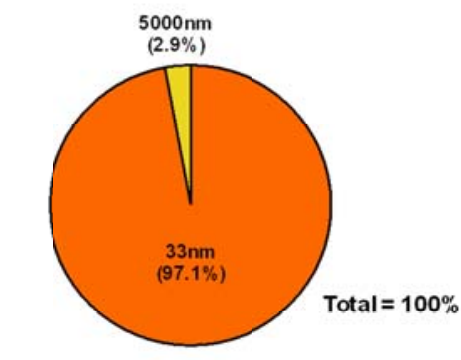

Peak 1 Mean $\square$ Peak 2 Mean

(c) Unfiltered Continuous CAI Processing

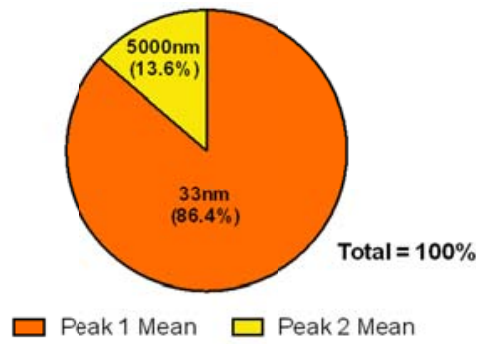

(b) Filtered Batch CAI Processing

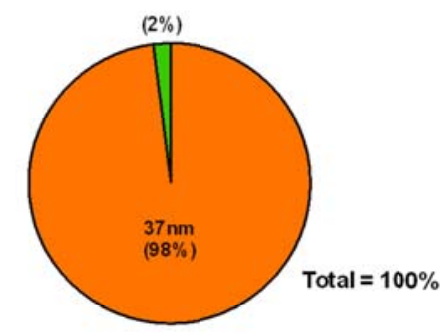

Peak1 Mean $\square$ CAILost

(d) Filtered Continuous CAI Processing

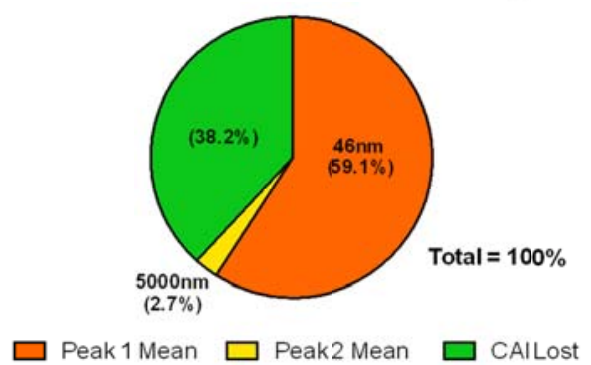

Figure 6. Pie charts showing the z-average values (in $\mathrm{nm}$ ) of the emulsions obtained at each peak and the percentage of emulsions with CAI obtained or lost (from filtration), in parenthesis, in each condition. Batch processing had a single step preparation with AFA treatment. Continuous processing allows all the production processes to occur without intervention. When CAI is dispersed evenly throughout oil droplet volume, (a) $97.1 \%$ of CAI encapsulated in $33 \mathrm{~nm}$ nanoemulsion, while $2.9 \%$ of CAI encapsulated in 5,000 nm microemulsion, for unfiltered batch CAI processing. (b) $98 \%$ of CAI was encapsulated in $37 \mathrm{~nm}$ nanoemulsion, while $2 \%$ of CAI was lost from filtering, for filtered batch CAI processing. (c) $86.4 \%$ of CAI was encapsulated in $33 \mathrm{~nm}$ nanoemulsion, while 13.6\% of CAI was encapsulated in 5,000 nm microemulsion, for unfiltered continuous CAI processing and (d) 59.1\% of CAI encapsulated in $46 \mathrm{~nm}$ nanoemulsion and $2.7 \%$ of CAI encapsulated in 5,000 nm microemulsion, while $38.2 \%$ of CAI was lost through filtration, in filtered continuous CAI processing.

For production needs, the flow rate may be multiplied if the desired final drug concentration is substantially below the maximum encapsulation concentration. To determine if a drug is suitable for scaling up in this manner, emulsions containing a range of drug concentrations should be produced in batch. Creaming in the samples is a simple way to determine if the concentration used is above saturation limit. HPLC analysis can be used on filtered batch samples to quantitatively determine the maximum encapsulation concentration. Maximum encapsulation concentration divided by necessary concentration times flow rate at encapsulation concentration provides the maximum production flow rate. For instance, the maximum encapsulation concentration for CAI in 4\% soybean oil, 4\% Tween-80 O/W emulsion is $2.4 \mathrm{mM}$. 
This emulsion yields particles of the appropriate size at $1 \mathrm{ml} / \mathrm{min}$. If the desired CAI concentration is $24 \mu \mathrm{M}$, with $0.04 \%$ soybean oil and $0.04 \%$ Tween- 80 , the highest production flow rate that can be achieved is $100 \mathrm{ml} / \mathrm{min}(2.4 \mathrm{mM} / 0.024 \mathrm{mM}$ $\times 1 \mathrm{ml} / \mathrm{min}=100 \mathrm{ml} / \mathrm{min}$ ). To produce nanoemulsions at this rate, a second Covaris S220x system should be set up immediately downstream of the first and run in series once the $1^{\text {st }}$ system has reached steady state. For the $2^{\text {nd }}$ system in the example above, the inlet composition should be $99 \mathrm{ml} / \mathrm{min} 3^{\circ} \mathrm{C}$ water and $1 \mathrm{ml} / \mathrm{min} 2.4 \mathrm{mM}$ CAI steady state nanoemulsion. System parameters of $100 \mathrm{~W}$ PIP, $50 \% \mathrm{DF}, 1,000 \mathrm{C} / \mathrm{B}, 3^{\circ} \mathrm{C}$ jacket temperature, and $3^{\circ} \mathrm{C}$ bath chiller temperature were found to promote mixing in the $2^{\text {nd }}$ system. This set up was tested without CAI and produced particles with the same size distribution as the $1^{\text {st }}$ system alone without increasing the ramp time (see Figure 7).

Figure 7. Blank nanoemulsions were produced without CAI in flow systems with and without a second S220X system added on the first system. The first system alone yielded nanoemulsions at $100 \times$ stock concentration. The second system produced nanoemulsions at the desired concentration. Z-average and pdI of the product stream were measured at varying time points and are plotted above.

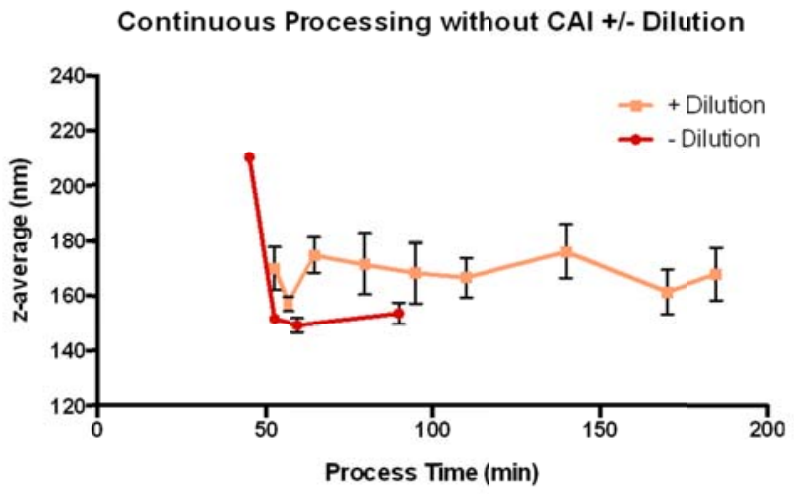

\section{Discussions}

One of the major challenges of the drug development has been achieving the effective bioavailability with tolerable drug concentration ${ }^{[38,39]}$. Many drugs experience difficulty in delivering appropriate dosage to a desired location and rapid renal filtration that requires frequent drug administration ${ }^{[7,27,40]}$. Nanoemulsion has been a great interest for pharmaceutical industries to address these problems. Not only it provides delayed renal filtration, but it also increases bioavailability to different locations, especially across the lipophilic barriers ${ }^{[4,8,27,31,41]}$. Despite the desired properties of nanoemulsions, the large-scale manufacturing has been difficult due to the limitations from the production processes. Requirement of large amount of solvents for the low-energy method and energy for the high-energy methods indicated the need of more economical methods of nanoemulsification ${ }^{[7,15]}$.

Ultrasonic emulsification was identified as the energy saving method among the currently available high-energy methods ${ }^{[15,30]}$. In order to address ultrasonication's limitation on preparing nanoemulsions with uniform and small sizes, Liquid Whistle Hydrodynamic Cavitation Reactor (LWHCR) has been developed. Coupling the characteristics of the ultrasonification with high-pressure homogenization and hydrodynamics, LWHCR seemed to improve on size distribution, stability, and scalability of the production ${ }^{[10,42]}$. However, both conventional ultrasonication (bath or probe form) and LWHCR is inadequate for large-scale production with economical energy consumption.

In this study, we have demonstrated that AFA technology can be a new method of producing nanoemulsions in a more economical and controlled manner. AFA's ability to focus the energy at a discrete location of the sample, unlike the bath ultrasonicator with unfocused energy delivery and therefore a lot of the energy is wasted in the water ${ }^{[3,34]}$, makes it very energy-efficient. In fact, AFA required about 163 times less power input than that of bath ultrasonicator to generate the same $1 \mathrm{MPa}$ pressure in the sample. At the same time, the temperature monitoring system in AFA allows the sample to maintain a desired temperature unlike the other conventional ultrasonic emulsification methods ${ }^{[43]}$. This introduces the possibility of preparing nanoemulsions with thermolabile therapeutic substances, such as proteins, which has been challenging due to the excessive heat generated from conventional methods. We have also shown that the delivered 
focused energy is sufficient to sterilize the solution during the preparation. This, followed by the filtration, would make the products highly sterile for the consumers.

Another key component for large-scale manufacturing is the system optimization, where various parameters of the instruments need to be set at the most plausible level for the highest quality products. The drugs used in this optimization process could be very costly. We have shown that the AFA produced nanoemulsions, with or without CAI, have similar sizes. This presents the capability of optimizing the nanoemulsification process ahead of time without consuming the therapeutic reagents to be encapsulated within the nanoemulsion. However, it is important to examine whether this observation holds for the larger molecules for wider application of the technology. We have also shown that PIP, flow rates, and jacket temperature of the process can influence the size of the nanoemulsions produced. With AFA technology's ability to acutely control each parameter through the instrument's unique software, it gives a reproducible and predictable production in a desired application. This understanding and control will be essential in improving the quality and efficiency of the pharmaceutical industry's manufacturing processes.

We have shown that the AFA technology enables the scale-up of the nanoemulsification process by allowing the transition from batch to continuous processing. CAI was effectively encapsulated in the nanoemulsions for both methods and it showed stability over time. The z-average of the nanoemulsions from continuous processing was higher than that of batch processing (see Table 2), and less CAI nanoemulsions were lost from the filtration of batch processing (see Figure 6). These observations may be resolved by further optimizing the parameters for continuous processing so that smaller nanoemulsions can be formed. We have also demonstrated that AFA system can be set up in series to increase the production flow rates, while maintaining similar nanoemulsion size as the singular setup. This result must be confirmed with the presence of drug, as our experiment was performed without CAI. However, the notion that multiple instruments setup in series can increase the flow rate offers a high-throughput strategy for the drug production using AFA technology.

AFA technology's ability to generate drug-encapsulating nanoemulsions in a continuous mode provides a potential for large-scale and cost-effective manufacturing processes for pharmaceutical industry. Moreover, it offers an opportunity to encapsulate and deliver thermolabile substances. These advantages will contribute in accelerating the manufacturing efforts of therapeutic nanoemulsions against various diseases.

\section{Conclusion}

This study demonstrates the ability of AFA technology to scale up production of a nanoemulsion containing a drug of pharmaceutical interest, CAI, from batch mode to continuous mode. Easily controlled factors, such as flow rate, temperature, composition, and AFA energy input, influence the nanoemulsion size distribution independently from the presence of drug, making the optimization simple and economical. Depending on a compound's maximum encapsulation concentration and the desired final concentration of the drug, producing the nanoemulsion using two Covaris' focused ultrasonicators in series could increase production rates substantially. AFA technology could be a realistic means of commercially producing a nanoemulsion encapsulating a hydrophobic drug.

\section{References}

[1] Solans C, Izquierdo P, Nolla J, et al. Nano-emulsions. Current Opinion in Colloid\&Interface Science. 2005; 10: 102-10. http://dx.doi.org/10.1016/j.cocis.2005.06.004

[2] Maali A, Mosavian MTH. Preparation and Application of Nanoemulsions in the last Decade (2000-2010). Journal of Dispersion Science and Technology. 2012; 34: 92-105. http://dx.doi.org/10.1080/01932691.2011.648498

[3] McClements DJ. Nanoemulsions Versus Microemulsions: Terminology, Differences, and Similarities. Soft Matter. 2012; 8: 1719-29. http://dx.doi.org/10.1039/C2SM06903B 
[4] Kotyla T, Kuo F, Moolchandani V, et al. Increased bioavailability of a transdermal application of a nano-sized emulsion preparation. International Journal of Pharmaceutics. 2008; 347: 144-8. http://dx.doi.org/10.1016/j.ijpharm.2007.06.045

[5] Shah P, Belodia D, Shelat P. Nanoemulsion: A Pharmaceutical Review. Systematic Review in Pharmacy. 2010; 1(1): 24-32. http://dx.doi.org/10.4103/0975-8453.59509

[6] Giri TK, Goswami NG, Jha VK. Prospective and Challenges of Micro-Emulsion as a Novel Carrier for Drug Delivery. Journal of Pharma Sci Tech. 2013; 2(2): 56-61.

[7] Lovelyn C, Attama AA. Current State of Nanoemulsions in Drug Delivery. Journal of Biomaterials and Nanobiotechnology. 2011; 2: 626-39. http://dx.doi.org/10.4236/jbnb.2011.225075

[8] Anton N, Benoit JP, Saulnier P. Design and Production of Nanoparticles Formulated from Nano-emulsion Templates - A review. Journal of Controlled Release. 2008; 128: 185-99. PMid:18374443. http://dx.doi.org/10.1016/j.jconrel.2008.02.007

[9] Shafiq S, Shakeel F, Talegaonkar S, et al. Development and Bioavailability Assessment of Ramipril Nanoemulsion Formation. European Journal of Pharmaceutics and Biopharmaceutics. 2007; 66: 227-43. PMid:17127045. http://dx.doi.org/10.1016/j.ejpb.2006.10.014

[10] Tang S, Sivakumar M. A novel and facile liquid whistle hydrodynamic cavitation reactor to produce submicron multiple emulsions. AlChE Journal. 2013; 59(1): 155-67. http://dx.doi.org/10.1002/aic.13800

[11] Deepak S, Gurmeet S, Mankaran S, et al. A Review on Innovative Approaches to Enhance Solubility and Dissolution Rate of Hydrophobic Drugs. Novel Science International Journal of Pharmaceutical Science. 2012; 1(7): 486-92.

[12] Drug-Carrier liposomes and Nanoemulsions. http://sonomechanics.com/applications/pharmaceutical/drug-carrier_liposomes_and_nanoemulsions/ (accessed Aug 5, 2014).

[13] Hedge RR, Verma A, Ghosh A. Microemulsion-New Insights into the Ocular Drug Delivery. ISRN Pharmaceuticals. 2013; 2013(2013): 1-11.

[14] Tang S, Sivakumar M, Ng A, et al. Anti-inflammatory and analgesic activity of novel oral aspirin-loaded nanoemulsion and nano multiple emulsion formulations generated using ultrasound cavitation. International Journal of Pharmaceutics. 2012; 430: 299-306. PMid:22503988. http://dx.doi.org/10.1016/j.ijpharm.2012.03.055

[15] Tang S, Shridharan P, Sivakumar M. Impact of process parameters in the generation of novel aspirin nanoemulsions-Comparative studies between ultrasound cavitation and microfluidizer. Ultrasonics Sonochemistry. 2013; 20: 485-97. PMid:22633626. http://dx.doi.org/10.1016/j.ultsonch.2012.04.005

[16] Floury J, Desrumaux A, Axelos MAV, et al. Degradation of Methylcellulose during Ultra-high Pressure Homogenization. Food Hydrocolloids. 2002; 16: 47-53. http://dx.doi.org/10.1016/S0268-005X(01)00039-X

[17] Gutierrez JM, Gonzalez C, Maestro A, et al. Nano-emulsions: New applications and optimization of their preparation. Current Opinion in Colloid and Interface Science. 2008; 13: 245-51. http://dx.doi.org/10.1016/j.cocis.2008.01.005

[18] Anton N, Gayet P, Benoit JP, et al. Nano-emulsions and nanocapsules by the PIT method: An investigation on the role of the temperature cycling on the emulsion phase inversion. International Journal of Pharmaceutics. 2007; 344: 44-52. PMid:17592746. http://dx.doi.org/10.1016/j.ijpharm.2007.04.027

[19] Tadros T, Izquierdo P, Esquena J, et al. Formation and stability of nano-emulsions. Advances in Colloid and Interface Science. 2004; 108-9: 303-18. PMid:15072948. http://dx.doi.org/10.1016/j.cis.2003.10.023

[20] Vandamme T, Anton N. Low-energy nanoemulsification to design veterinary controlled drug delivery devices. International Journal of Nanomedicine. 2010; 5: 867-73. PMid:21042549. http://dx.doi.org/10.2147/IJN.S13273

[21] Peshkovsky AS, Bystryak S. Continuous-flow Production of a Pharmaceutical Nanoemulsion by High Amplitude Ultrasound: Process Scale-Up. Chemical Engineering and Processing: Process Intensification. 2014; 82: 132-6. http://dx.doi.org/10.1016/j.cep.2014.05.007

[22] Zeeb B, Herz E, McClements D, et al. Impact of alcohols on the formation and stability of protein-stabilized nanoemulsions. Journal of Colloid and Interface Science. 2014; 433: 196-203. PMid:25129338. http://dx.doi.org/10.1016/j.jcis.2014.07.034

[23] Dhat S, Pund S, Kokare C. et al. Mechanistic investigation of biopharmaceutic and pharmacokinetic characteristics of surface engineering of satranidazole nanocrystals. European Journal of Pharmaceutics and Biopharmaceutics. 2016. PMid:26748382. http://dx.doi.org/10.1016/j.ejpb.2015.12.007

[24] Date A, Desai N, Dixit R, et al. Self-nanoemulsifying drug delivery systems: formulation insights, applications and advances. Nanomedicine. 2010; 5(10): 1595-616. PMid:21143036. http://dx.doi.org/10.2217/nnm.10.126

[25] Jafari SM, He Y, Bhandari B. Nano-emulsion Production by Sonication and Microfluidization-A Comparison. International Journal of Food Properties. 2006; 9: 475-85. http://dx.doi.org/10.1080/10942910600596464

[26] Vladisavljevic GT, Kobayashi I. Production of uniform droplets using membrane, microchannel and microfluidic emulsification devices. Microfluid Nanofluid. 2012; 13: 151-78. http://dx.doi.org/10.1007/s10404-012-0948-0 
[27] Hickey J, Santos J, Williford JM, et al. Control of polymeric nanoparticle size to improve therapeutic delivery. Journal of Controlled Release. 2015; 219: 536-47. PMid:26450667. http://dx.doi.org/10.1016/j.jconrel.2015.10.006

[28] Bai L, McClements D. Development of microfluidization methods for efficient production of concentrated nanoemulsions: Comparison of single- and dual-channel microfluidizers. Journal of Colloid and Interface Science. 2016; 466: $206-12$. PMid:26724703. http://dx.doi.org/10.1016/j.jcis.2015.12.039

[29] Canselier JP, Delmas H, Wilhelm AM, et al. Ultrasound Emulsification-An Overview. Journal of Dispersion Science and Technology. 2002; 23(1-3): 333-49. http://dx.doi.org/10.1080/01932690208984209

[30] Tang S, Manickam S, Wei T, et al. Formulation development and optimization of a novel Cremophore EL-based nanoemulsion using ultrasound cavitation. Ultrasonics Sonochemistry. 2012; 19: 330-45. PMid:21835676. http://dx.doi.org/10.1016/j.ultsonch.2011.07.001

[31] Lopes M, Abrahim-Vieira B, Oliveira C, et al. Probing insulin bioactivity in oral nanoparticles produced by ultrasonicationassisted emulsification/internal gelation. International Journal of Nanomedicine. 2015; 10: 5865-80. PMid:26425087.

[32] Tang S, Sivakumar M, Nashiru B. Impact of osmotic pressure and gelling in the generation of highly stable single core water-in-oil-in-water (W/O/W) nano multiple emulsions of aspirin assisted by two-stage ultrasonic cavitational emulsification. Colloids and Surfaces B: Biointerfaces. 2013; 102: 653-8. PMid:23107943. http://dx.doi.org/10.1016/j.colsurfb.2012.08.036

[33] Nascentes C, Korn M, Sousa C, et al. Use of Ultrasonic Baths for Analytical Applications: A New Approach for Optimisation Conditions. Journal of Brazilian Chemical Society. 2001; 12(1): 57-63. http://dx.doi.org/10.1590/S0103-50532001000100008

[34] Capote F, Castro L. ANALYTICAL APPLICATIONS OF ULTRASOUND. Elsevier. 2007: 12-27.

[35] Adjei I, Peetla C, Labhasetwar V. Heterogeneity in nanoparticles influences biodistribution and targeting. Nanomedicine. 2014; 9(2): 267-78. PMid:23799984. http://dx.doi.org/10.2217/nnm.13.70

[36] Luzzi KJ, Varghese HJ, Macdonald IC, et al. Inhibition of angiogenesis in liver metastases by carboxyamidotriazole (CAI). Angiogenesis. 1998; 2(4): 373-9. PMid:14517457. http://dx.doi.org/10.1023/A:1009259521092

[37] Dynamic Light Scattering: Common Terms Defined. University of Cambridge Department of Biochemistry Website. 2011. http://www.biophysics.bioc.cam.ac.uk/wp-content/uploads/2011/02/DLS_Terms_defined_Malvern.pdf (accessed Aug 13, 2014).

[38] Anand P, Kunnumakkara A, Newman R, et al. Bioavailability of Curcumin: Problems and Promises. 2007; 4(6): 807-18.

[39] Tiwari G, Tiwari R, Sriwastawa B, et al. Drug delivery systems: An updated review. International Journal of Pharmaceutical Investigation. 2012; 2(1): 2-11. PMid:23071954. http://dx.doi.org/10.4103/2230-973X.96920

[40] Jaiswal M, Dudhe R, Sharma P. Nanoemulsion: an advanced mode of drug delivery system. Biotech. 2015; 5(2): 123-7. http://dx.doi.org/10.1007/s13205-014-0214-0

[41] Fornaguera C, Dols-Perez A, Caldero G, et al. PLGA nanoparticles prepared by nano-emulsion templating using low-energy methods as efficient nanocarriers for drug delivery across the blood-brain barrier. Journal of Controlled Release. 2015; 211: 134-43. PMid:26057857. http://dx.doi.org/10.1016/j.jconrel.2015.06.002

[42] Parthasarathy S, Ying T, Manickam S. Generation and Optimization of Palm Oil-Based Oil-in-Water (O/W) SubmicronEmulsions and Encapsulation of Curcumin Using a Liquid Whistle Hydrodynamic Cavitation Reactor (LWHCR). Industrial and Engineering Chemistry Research. 2013; 52: 11829-37. http://dx.doi.org/10.1021/ie4008858

[43] Kakumanu S, Schroeder A. Focused Ultrasound - A Novel Tool for Liposome Formulation. Drug Development \& Delivery. 2012; 12(5): 47-52. 